\title{
STRUCTURE-PRESERVING MODEL REDUCTION USING A KRYLOV SUBSPACE PROJECTION FORMULATION*
}

\author{
REN-CANG $\mathrm{LI}^{\dagger}$ AND ZHAOJUN BAI ${ }^{\ddagger}$
}

\begin{abstract}
A general framework for structure-preserving model reduction by Krylov subspace projection methods is developed. It not only matches as many moments as possible but also preserves substructures of importance in the coefficient matrices $L, G, C$, and $B$ that define a dynamical system prescribed by the transfer function of the form $H(s)=L^{*}(G+s C)^{-1} B$. Many existing structurepreserving model-order reduction methods for linear and second-order dynamical systems can be derived under this general framework. Furthermore, it also offers insights into the development of new structure-preserving model reduction methods.
\end{abstract}

Key words. model reduction, structure-preserving, moment matching, Krylov subspace, Arnoldi process, Lanczos process.

AMS subject classifications. 65F15, 65F50, 15A18, 37M05

\section{Introduction}

Krylov subspace projection methods are increasingly popular in model reduction owing to their numerical efficiency for very large systems, such as those arising from structure dynamics, control systems, circuit simulations, computational electromagnetics and microelectromechanical systems $[13,10,12,5,42,47,48,52]$. Recent survey articles $[1,4,19]$ provide in depth review of the subject and comprehensive references. Roughly speaking, these methods project the original state-space onto a low-dimensional subspace to arrive at a (much) smaller system having properties, among others, that many leading terms (called moments) of the associated (matrixvalued) transfer functions expanded at given points for the original and reduced systems match.

Consider the matrix-valued transfer function of the form

$$
H(s)=L^{*}(G+s C)^{-1} B,
$$

which describes an associated multi-input multi-output (MIMO) time-invariant system to be studied. Here $G, C \in \mathbb{C}^{N \times N}, B \in \mathbb{C}^{N \times m}, L \in \mathbb{C}^{N \times p}$. The power series expansion of $H(s)$ at $s=0$ is formally given by, assuming $G$ is nonsingular,

$$
H(s)=\sum_{i=0}^{\infty}(-1)^{i} s^{i} M_{i}
$$

where moments $M_{i}$ are defined as

$$
M_{i} \stackrel{\text { def }}{=} L^{*}\left(G^{-1} C\right)^{i} G^{-1} B \equiv L^{*} G^{-1}\left(C G^{-1}\right)^{i} B
$$

In today's applications of interest, such as VLSI circuit designs and structural dynamics, $N$ can be up to millions $[1,4,20]$. Computations of $H(s)$ usually have to be

\footnotetext{
${ }^{*}$ Received: February 9, 2005; accepted (in revised version): April 4, 2005. Communicated by Shi Jin.

${ }^{\dagger}$ Department of Mathematics, University of Kentucky, Lexington, KY 40506, USA (rcli@ms. uky.edu).

${ }_{\ddagger}$ Department of Computer Science and Department of Mathematics, University of California, Davis, CA 95616, USA (bai@cs.ucdavis.edu).
} 
done through some kind of reduction on $L, G, C$ and $B$. Let $X, Y \in \mathbb{C}^{N \times n}$ such that $Y^{*} G X$ is nonsingular (and thus $\operatorname{rank}(X)=\operatorname{rank}(Y)=n$ ). We may reduce the transfer function $H(s)$ to

$$
H_{\mathrm{R}}(s)=L_{\mathrm{R}}^{*}\left(G_{\mathrm{R}}+s C_{\mathrm{R}}\right)^{-1} B_{\mathrm{R}},
$$

where

$$
L_{\mathrm{R}}=X^{*} L, G_{\mathrm{R}}=Y^{*} G X, C_{\mathrm{R}}=Y^{*} C X, B_{\mathrm{R}}=Y^{*} B .
$$

The associated moments of $H_{\mathrm{R}}(s)$ at $s=0$ are

$$
M_{\mathrm{R} i} \stackrel{\text { def }}{=} L_{\mathrm{R}}^{*}\left(G_{\mathrm{R}}^{-1} C_{\mathrm{R}}\right)^{i} G_{\mathrm{R}}^{-1} B_{\mathrm{R}} \equiv L_{\mathrm{R}}^{*} G_{\mathrm{R}}^{-1}\left(C_{\mathrm{R}} G_{\mathrm{R}}^{-1}\right)^{i} B_{\mathrm{R}},
$$

for $i=0,1,2, \ldots$. There are various techniques to pick $X$ and $Y$ to perform reduction. Among them Krylov subspace-based model-reduction is getting much of the attention. The idea is to pick $X$ and $Y$ as the bases of properly defined Krylov subspaces to match as many $M_{i}$ to $M_{\mathrm{R} i}$ as possible from $i=0$ and forward. If $M_{i}=M_{\mathrm{R} i}$ for $0 \leq i \leq \ell$, then $H(s)=H_{\mathrm{R}}(s)+\mathcal{O}\left(s^{\ell+1}\right)$.

The general form (1.1) is often treated by rewriting it as, e.g.,

$$
H(s)=L^{*}\left(I+s G^{-1} C\right)^{-1}\left(G^{-1} B\right) .
$$

By doing so $G^{-1} C$ is projected as one whole matrix, unlike above where $G$ and $C$ are projected separately. See $[16,23,41]$. There are advantages and disadvantages associated with the two different ways to do projections. The advantage would be some computational saving because the projection of $G^{-1} C$, namely $Y^{*} G^{-1} C X$, is often computed while bases of Krylov subspaces are being built, regardless of how $G$ and $C$ are projected: separately or as a whole in terms of $G^{-1} C$, and so extra work is needed if $G$ and $C$ are projected separately. The disadvantages include the loss of structures in $G$ and $C$, such as symmetry and possibly stabilities inherent in the original full-order systems. One prime example would be PRIMA [38] which preserves the important stability property and passivity of the original system from LRC circuits. Recently, researchers are more inclined to project $G$ and $C$ separately $[49,38,55,24,7,8,18,50]$ because of its capability of structure preservations.

For the case of $C=I$ (and thus $Y^{*} X=I_{n}$ ), Villemagne and Skelton [51, 1987] gave a thorough study of the conditions on $X$ and $Y$ under which the number of moments match, and arrived at many results, some new and some old, dated back as early as $[27,1974]$. These results consist of the foundation of the PVL algorithm [16] and a similar one [23] proposed in the mid 1990s to provide a more stable implementation of the asymptotic waveform evaluation algorithm [41]. For the case of $C \neq I$, a general moment-match theory was developed by Grimme [28]. This paper will exploit these existing moment-matching theorems, such as those presented in [51, 28], to design an algorithmic framework that not only matches moments, but also preserves substructures in the coefficient matrices $L, G, C$ and $B$. We show that many existing structure-preserving methods, such as the ones presented in $[49,38,7]$ for linear and second-order dynamical systems, can all be derived under this general framework.

This paper is based on two recent technical reports of the authors [33, 34], and part of the results has been reported in the conference proceeding [35].

The rest of this paper is organized as follows. Section 2 defines two general projectors that will be used to prove moment-matching theorems in Section 3 in a 
simpler way. These moment-matching theorems are the theoretical foundation of this paper. A general framework to design structure preserving model reduction is given in Section 4, and its application to the transfer function of a second-order system is in Section 6 with the help of the inherent structural properties of Krylov subspaces for certain block matrices presented in Section 5 .

Throughout the paper, $\mathbb{C}, \mathbb{C}^{k}$, and $\mathbb{C}^{k \times \ell}$ are the sets of complex numbers, column vectors of dimension $k$, and $k \times \ell$ complex matrices, respectively. $I_{k} \in \mathbb{C}^{k \times k}$ is the identity matrix, and sometimes simply $I$ when its dimension can be judged from the context. Unless otherwise explicitly stated, capital letters are matrices, while lower case letters are vectors or scalars. $X^{*}$ is the complex conjugate transpose of matrix $X$, $\operatorname{span}(X)$ is the subspace spanned by the columns of $X$ with dimension $\operatorname{dim}(\operatorname{span}(X))=$ $\operatorname{rank}(X)$, the rank of $X$. If span takes more than one argument matrices, it is the subspace spanned by all the columns of those matrices combined. For scalar $\alpha, \bar{\alpha} \stackrel{\text { def }}{=} \alpha^{*}$.

Let $A$ be $N \times N$, and let $Z$ be $N \times \ell$. The kth Krylov subspace generated by $A$ on $Z$ is defined to be

$$
\mathcal{K}_{k}(A, Z)=\operatorname{span}\left(Z, A Z, \ldots, A^{k-1} Z\right) .
$$

For convenience, when $k=0$, define $\mathcal{K}_{0}(A, Z)=\{0\}$, a trivial subspace.

\section{Projectors}

The use of projection techniques is a classical and powerful idea. It is an indispensable tool in studying infinite dimensional operator theory, but one may be able to get around it in finite dimensional cases through matrix manipulations, for example, the moment-matching proofs in $[6,9,19,38,49,53]$. However, using projection language can turn those proofs into much more elegant mathematical arguments as already made clear by [51].

Any matrix (operator) $P$ that satisfies $P^{2}=P$ is a projector onto $\operatorname{span}(P)$. Let $k=\operatorname{rank}(P)$. We have (for example, through its singular value decomposition (SVD) $[15,26])$

$$
P=X \Sigma Y^{*},
$$

where $X, Y \in \mathbb{C}^{N \times n}$ with $\operatorname{rank}(X)=\operatorname{rank}(Y)=n$, and $\Sigma \in \mathbb{C}^{n \times n}$ nonsingular. $P^{2}=P$ implies $Y^{*} X$ is nonsingular and $\Sigma=\left(Y^{*} X\right)^{-1}$. Then

$$
P=X\left(Y^{*} X\right)^{-1} Y^{*} .
$$

It can be verified that any matrix of this form satisfies $P^{2}=P$. Thus (2.1) gives all projectors of rank $n$. It can also be seen that $P$ projects onto $\operatorname{span}(X)=\operatorname{span}(P)$ which means $P x=x$ for any $x \in \operatorname{span}(X)$ and especially $P X=X$. On the other hand, $P^{*}$ projects onto $\operatorname{span}(Y)$ and thus $y^{*} P=y^{*}$ for any $y \in \operatorname{span}(Y)$ and especially $Y^{*} P=Y^{*}$.

The following two seemingly more general but equivalent forms for projectors of rank $n$ :

$$
\begin{aligned}
& P=X\left(Y^{*} G X\right)^{-1} Y^{*} G, \\
& Q=G X\left(Y^{*} G X\right)^{-1} Y^{*},
\end{aligned}
$$

will be useful in the sequel. Here $G$ is an $N \times N$ matrix such that $Y^{*} G X$ is nonsingular. Notice that both $P$ in (2.2) and $Q$ in (2.3) become the $P$ in (2.1) when $G=I$. The introduction of $G$ will become handy for us later. The following lemma is well-known, 
and it shows that $P$ projects onto $\operatorname{span}(X)$ and $Q^{*}$ projects onto $\operatorname{span}(Y)[31$, Page $20]$.

Lemma 2.1. Let projectors $P$ and $Q$ be defined as in (2.2) and (2.3). Then

$$
\begin{aligned}
P x & =x, \quad \text { for any } x \in \operatorname{span}(X) \\
y^{*} Q & =y^{*}, \quad \text { for any } y \in \operatorname{span}(Y) .
\end{aligned}
$$

\section{Moment-matching theorems}

Let notation from (1.1) to (1.5) have their assignments there. Villemagne and Skelton [51] and Grimme [28] developed a general theorem governing the number of moments matched as we will present in this section. However, we shall give a different proof using the projectors $P$ and $Q$ in Section 2. The keys are the following two lemmas. The proof seems a bit more concise. Grimme's proof was not explicitly formulated in terms of $P$ and $Q$, but rather implicitly.

Lemma 3.1. Let projector $P$ be defined as in (2.2). Then

$$
X G_{\mathrm{R}}^{-1} B_{\mathrm{R}}=P G^{-1} B \quad \text { and } \quad X G_{\mathrm{R}}^{-1} C_{\mathrm{R}}=P G^{-1} C \cdot X .
$$

Together they imply

$$
X\left(G_{\mathrm{R}}^{-1} C_{\mathrm{R}}\right)^{i} G_{\mathrm{R}}^{-1} B_{\mathrm{R}}=\left(P G^{-1} C\right)^{i} \cdot P G^{-1} B \quad \text { for } i \geq 0 .
$$

Proof. By (1.4), we have

$$
X G_{\mathrm{R}}^{-1} B_{\mathrm{R}}=X\left(Y^{*} G X\right)^{-1} \cdot Y^{*} G G^{-1} B=P G^{-1} B,
$$

and

$$
X G_{\mathrm{R}}^{-1} C_{\mathrm{R}}=X\left(Y^{*} G X\right)^{-1} \cdot Y^{*} G G^{-1} C X=P G^{-1} C \cdot X
$$

as expected.

Lemma 3.2. Let projector $Q$ be defined as in (2.3). Then

$$
L_{\mathrm{R}}^{*} G_{\mathrm{R}}^{-1} Y^{*}=L^{*} G^{-1} Q \quad \text { and } \quad C_{\mathrm{R}} G_{\mathrm{R}}^{-1} Y^{*}=Y^{*} \cdot C G^{-1} Q .
$$

Together they imply

$$
L_{\mathrm{R}}^{*} G_{\mathrm{R}}^{-1}\left(C_{\mathrm{R}} G_{\mathrm{R}}^{-1}\right)^{i} Y^{*}=L^{*} G^{-1} Q \cdot\left(C G^{-1} Q\right)^{i} \quad \text { for } i \geq 0 .
$$

Proof. Notice that projector $P$ in (2.2) gives rise to projector $Q$ in (2.3) under substitutions

$$
X \rightarrow Y, \quad Y \rightarrow X, \quad G \rightarrow G^{*}
$$

followed by taking conjugate transpose. In view of this, with two more substitutions

$$
B \rightarrow L, C \rightarrow C^{*},
$$


in additional to those in (3.2), we obtain (3.1) by Lemma 3.1 .

REMARK 3.1. It is worth noting that Lemmas 3.1 and 3.2 assume only the invertibility of $Y^{*} G X$, and they hold regardless of whatever $\operatorname{span}(X)$ and $\operatorname{span}(Y)$ are.

Theorem 3.3. ([51, 28]) Let integers $k, r \geq 0$. If

$$
\mathcal{K}_{k}\left(G^{-1} C, G^{-1} B\right) \subseteq \operatorname{span}(X) \quad \text { and } \quad \mathcal{K}_{r}\left(G^{-*} C^{*}, G^{-*} L\right) \subseteq \operatorname{span}(Y),
$$

then

$$
X\left(G_{\mathrm{R}}^{-1} C_{\mathrm{R}}\right)^{i} G_{\mathrm{R}}^{-1} B_{\mathrm{R}}=\left(G^{-1} C\right)^{i} G^{-1} B \quad \text { for } 0 \leq i \leq k-1
$$

and

$$
L_{\mathrm{R}}^{*} G_{\mathrm{R}}^{-1}\left(C_{\mathrm{R}} G_{\mathrm{R}}^{-1}\right)^{j} Y^{*}=L^{*} G^{-1}\left(C G^{-1}\right)^{j} \quad \text { for } 0 \leq j \leq r-1 .
$$

Together they imply that

$$
M_{i}=M_{\mathrm{R} i} \quad \text { for } 0 \leq i \leq k+r-1 .
$$

Proof. Let projectors $P$ and $Q$ be defined as in (2.2) and (2.3). Then (3.4) and Lemma 2.1 yield

$$
\begin{aligned}
P\left(G^{-1} C\right)^{i} G^{-1} B & =\left(G^{-1} C\right)^{i} G^{-1} B & & \text { for } 0 \leq i \leq k-1, \\
L^{*} G^{-1}\left(C G^{-1}\right)^{j} Q & =L^{*} G^{-1}\left(C G^{-1}\right)^{j} & & \text { for } 0 \leq j \leq r-1 .
\end{aligned}
$$

Equality (3.5) is now a consequence of (3.8) and Lemma 3.1. Similarly, equality (3.6) is a consequence of (3.9) and Lemma 3.2. Now for $0 \leq i \leq k-1$ and $0 \leq j \leq r-1$ we have

$$
\begin{aligned}
M_{\mathrm{R} i+j+1} & =L_{\mathrm{R}}^{*} G_{\mathrm{R}}^{-1}\left(C_{\mathrm{R}} G_{\mathrm{R}}^{-1}\right)^{j} C_{\mathrm{R}}\left(G_{\mathrm{R}}^{-1} C_{\mathrm{R}}\right)^{i} G_{\mathrm{R}}^{-1} B_{\mathrm{R}} \\
& =L_{\mathrm{R}}^{*} G_{\mathrm{R}}^{-1}\left(C_{\mathrm{R}} G_{\mathrm{R}}^{-1}\right)^{j} Y^{*} \cdot C \cdot X\left(G_{\mathrm{R}}^{-1} C_{\mathrm{R}}\right)^{i} G_{\mathrm{R}}^{-1} B_{\mathrm{R}} \\
& =L^{*} G^{-1}\left(C G^{-1}\right)^{j} \cdot C \cdot\left(G^{-1} C\right)^{i} G^{-1} B \\
& =M_{i+j+1} .
\end{aligned}
$$

Finally $M_{\mathrm{R} 0}=L_{\mathrm{R}}^{*} G_{\mathrm{R}}^{-1} B_{\mathrm{R}}=L^{*} X G_{\mathrm{R}}^{-1} B_{\mathrm{R}}=L^{*} G^{-1} B=M_{0}$. This completes the proof. $\square$

Corollary 3.4. Assume that $G$ and $C$ are Hermitian. If

$$
\mathcal{K}_{q}\left(G^{-1} C, G^{-1}(B L)\right) \subseteq \operatorname{span}(X)
$$

and $Y=X$, then $M_{i}=M_{\mathrm{R} i}$ for $0 \leq i \leq 2 k-1$.

Proof. $Y=X$ and (3.10) imply (3.4) with $r=q$. a shift

In case when approximations to $H(s)$ around a selected point $s_{0} \neq 0$ are sought,

$$
s=s_{0}+\left(s-s_{0}\right)
$$

can be performed and then

$$
G+s C=G+s_{0} C+\left(s-s_{0}\right) C \stackrel{\text { def }}{=} G\left(s_{0}\right)+\widetilde{s} C .
$$


Upon substitutions (i.e., renaming)

$$
G\left(s_{0}\right) \rightarrow G, \quad \widetilde{s} \rightarrow s,
$$

the problem of approximating $H(s)$ around $s=s_{0}$ becomes equivalent to approximating the substituted $H(s)$ around $s=0$. Observe that any reduction on $G\left(s_{0}\right)$ and $C$ by $Y^{*} G\left(s_{0}\right) X$ and $Y^{*} C X$ can be done through reducing $G$ and $C$ directly as in (1.4) because

$$
G_{\mathrm{R}}\left(s_{0}\right) \stackrel{\text { def }}{=} Y^{*} G\left(s_{0}\right) X=Y^{*} G X+s_{0} Y^{*} C X=G_{\mathrm{R}}+s_{0} C_{\mathrm{R}} .
$$

This is a significant observation because it says that even for approximating $H(s)$ near a different point $s_{0} \neq 0$, reduction can still be done directly to the original matrices $L$, $G, C$, and $B$, regardless of the shift (3.11). This will become handy when substructures in the original system are worth preserving.

As a straightforward application of Theorem 3.3, we have the following theorem, which can be viewed as a special case of Grimme's theorem in [28, Theorem 3.1].

TheOREm 3.5. Let integers $k, r \geq 0$, and let $G\left(s_{0}\right)$ be defined as in (3.12). If

$$
\mathcal{K}_{k}\left(G\left(s_{0}\right)^{-1} C, G\left(s_{0}\right)^{-1} B\right) \subseteq \operatorname{span}(X)
$$

and

$$
\mathcal{K}_{r}\left(G\left(s_{0}\right)^{-*} C^{*}, G\left(s_{0}\right)^{-*} L\right) \subseteq \operatorname{span}(Y),
$$

then

$$
M_{i}\left(s_{0}\right)=M_{\mathrm{R} i}\left(s_{0}\right) \quad \text { for } 0 \leq i \leq k+r-1,
$$

where $\quad M_{i}\left(s_{0}\right) \stackrel{\text { def }}{=} L^{*}\left(G\left(s_{0}\right)^{-1} C\right)^{i} G\left(s_{0}\right)^{-1} B \quad$ and $\quad M_{\mathrm{R} i}\left(s_{0}\right) \stackrel{\text { def }}{=}$ $L_{\mathrm{R}}^{*}\left(G_{\mathrm{R}}\left(s_{0}\right)^{-1} C_{\mathrm{R}}\right)^{i} G_{\mathrm{R}}\left(s_{0}\right)^{-1} B_{\mathrm{R}} . \quad$ This implies $H(s)=H_{\mathrm{R}}(s)+\mathcal{O}\left(\left(s-s_{0}\right)^{k+r}\right)$.

REMARK 3.2. The invariance property (3.13) of the reduction on $L, G, C$, and $B$ regardless of the shift (3.11) makes it possible to match moments at multiple points by one reduction. This is done by enforcing $\operatorname{span}(X)$ and/or $\operatorname{span}(Y)$ containing more appropriate Krylov subspaces associated at multiple points. To avoid repetition, we shall omit explicitly stating it. See [28] and Ruhe [43, 44].

REMARK 3.3. Other theorems for at $s_{0}=0$ in later sections have analogous counterparts for at $s_{0} \neq 0$ too. We shall avoid explicitly stating them.

In the rest of this section, we shall apply Theorem 3.3 to three well-known methods as examples to show the generality of these moment-matching theorems. Application to the partial Padé-via-Lanczos method [6] is not obvious (see Example 3.3). Two more applications are in the later sections - one is to devise a technique that makes it possible to preserve block substructures in the model matrices $L, G, C$ and $B$, and the other is on the transfer the function of second-order dynamical systems.

EXAMPLE 3.1. When $G=I$ and $p=m=1, H(s)$ and $H_{\mathrm{R}}(s)$ are usually written as, assuming $Y^{*} X=I_{n}$,

$$
H(s)=l^{*}(I+s A)^{-1} b \quad \text { and } \quad H_{\mathrm{R}}(s)=l_{\mathrm{R}}^{*}\left(I+s A_{\mathrm{R}}\right)^{-1} b_{\mathrm{R}}
$$


and the associated moments are $M_{i}=l^{*} A^{i} b$ and $M_{\mathrm{R} i}=l_{\mathrm{R}}^{*} A_{\mathrm{R}}^{i} b_{\mathrm{R}}$. If

$$
\operatorname{span}(X)=\mathcal{K}_{k}(A, b) \quad \text { and } \operatorname{span}(Y)=\mathcal{K}_{k}\left(A^{*}, l\right),
$$

then Theorem 3.3 implies $M_{i}=M_{\mathrm{R} i}$ for $0 \leq i \leq 2 k-1, H_{\mathrm{R}}(s)$ is a Padé approximation of $H(s)$. The idea of the algorithms presented in $[16,23]$ is to run non-symmetric Lanczos [32] to generate bi-orthogonal bases for $\mathcal{K}_{k}(A, b)$ and $\mathcal{K}_{k}\left(A^{*}, l\right)$ and at the same time produce the reduced $l_{\mathrm{R}}, A_{\mathrm{R}}, b_{\mathrm{R}}$, a process that is much more stable than the mathematically equivalent asymptotic waveform evaluation [41]. Of course nonsymmetric Lanczos can run into stability problems and breakdowns of its own as well. Details along these lines are out of the scope of this paper. Interested readers may read, e.g., [40, 39, 22, 54, 3].

If only one of the two conditions in (3.14) is made true, e.g., $\operatorname{span}(X)=\mathcal{K}_{k}(A, b)$ by Arnoldi process [2] and $Y=X$ or any $N \times n$ matrix such that $Y^{*} X=I_{n}$, then $M_{i}=M_{\mathrm{R} i}$ for only $0 \leq i \leq k-1$ in general.

There is a straightforward block version of this example when $m \neq 1$ and/or $p \neq 1$. Depending on whether $r=0$ or not, either a block Arnoldi process or a block nonsymmetric Lanczos process can be used to generate $X$ and $Y$.

EXAMPLE 3.2. The PRIMA algorithm considers the transfer function $H(s)$ where $L=B[38]$. By taking $Y=X$ and

$$
\operatorname{span}(X)=\mathcal{K}_{k}\left(G^{-1} C, G^{-1} B\right),
$$

Theorem 3.3 implies that $M_{i}=M_{\mathrm{R} i}$ for $0 \leq i \leq k-1$. The proof in [38] did not use the language of projection. The columns of $X$ which form an orthonormal basis of the Krylov subspace is usually computed by the Arnoldi process [2] if $B$ is a vector or its natural block version extension otherwise.

As is, $G$ in the formulation in PRIMA [38] is not Hermitian, while $C$ is. Nevertheless a simple sign change can make both $G$ and $C$ Hermitian; see [21] or Section 4. A benefit of doing so is given in Corollary 3.4, especially when $L=B$ which is the case in the formulation of SyMPVL method [21].

EXAMPLE 3.3. This is for the moment-matching of a partial Padé-via-Lanczos method presented in [6]. As in Example 3.1, it concerns the SISO transfer functions

$$
H(s)=l^{*}(I+s A)^{-1} b \quad \text { and } \quad H_{\mathrm{R}}(s)=l_{\mathrm{R}}^{*}\left(I+s A_{\mathrm{R}}\right)^{-1} b_{\mathrm{R}} .
$$

After $k$-steps, assuming no breakdowns, the non-symmetric Lanczos procedure generates right and left Lanczos vectors

$$
v_{1}, v_{2}, \ldots, v_{k+1} \quad \text { and } w_{1}, w_{2}, \ldots, w_{k+1}
$$

where $v_{1}=\alpha b$ and $w_{1}=\beta l$ for some $\alpha \neq 0 \neq \beta$. Let $V_{j}=\left(\begin{array}{llll}v_{1} & v_{2} & \cdots & v_{j}\end{array}\right)$ and $W_{j}=$ $\left(\begin{array}{llll}w_{1} & w_{2} & \cdots & w_{j}\end{array}\right)$. Then ${ }^{1} W_{j}^{*} V_{j}=I_{j}$ for $1 \leq j \leq k, W_{k}^{*} v_{k+1}=0=V_{k}^{*} w_{k+1}$, and

$$
\operatorname{span}\left(V_{j}\right)=\mathcal{K}_{j}(A, b), \quad \operatorname{span}\left(W_{j}\right)=\mathcal{K}_{j}\left(A^{*}, l\right) .
$$

Thus let $X=V_{k}$ and $Y=W_{k}$; by Theorem 3.3, we have

$$
H_{\mathrm{R}}(s)=\left(l^{*} b\right) e_{1}^{*}\left(I+s T_{k}\right)^{-1} e_{1}=H(s)+\mathcal{O}\left(s^{2 k}\right),
$$

\footnotetext{
${ }^{1} \mathrm{Bi}$-orthogonalization between $v_{k+1}$ and $w_{k+1}$ has not been done yet, i.e., $w_{k+1}^{*} v_{k+1}$ can be anything, including zero. Thus possible breakdown may occur at the very next Lanczos step.
} 
where $T_{k}=W_{k}^{*} A V_{k}$. But this $H_{\mathrm{R}}(s)$ as is may be unstable in the sense that $T_{k}$ has eigenvalues in the right half plane of $\mathbb{C}$ while the original $A$ does not. Bai and Freund [6] proposed to update $T_{k}$ by a rank-one matrix $z e_{k}^{*}$ to $T_{k}+z e_{k}^{*}$ and hopefully with a judicial choice of the vector $z \in \mathbb{C}^{k}, T_{k}+z e_{k}^{*}$ has no eigenvalues in the right half plane, and accordingly used $\widetilde{H}_{\mathrm{R}}(s)=\left(l^{*} b\right) e_{1}^{*}\left[I+s\left(T_{k}+z e_{k}^{*}\right)\right]^{-1} e_{1}$ as a reduced model. By carefully exploiting the zero structures in $T_{k}+z e_{k}^{*}$, they proved if $z$ 's entries, except its last $\ell$ ones, are zeros, then

$$
\widetilde{H}_{\mathrm{R}}(s)=H(s)+\mathcal{O}\left(s^{2 k-\ell}\right) .
$$

We now apply Theorem 3.3 to reach the same conclusion. Notice that the motivation of employing the rank-1 update makes the situations $v_{k+1}=0$ or $w_{k+1}=0$ uninteresting because either of the two implies all eigenvalues of $T_{k}$ are $A$ 's. Nevertheless the proof below requires that only one of the two not be zero. Assume $v_{k+1} \neq 0$. Take $Y=W_{k}+w z^{*}$ and $X=V_{k}$, where $w$ is any vector such that $V_{k+1}^{*} w=e_{k+1}$. Such $w$ exists because $\operatorname{rank}\left(V_{k+1}\right)=k+1$. It is known that $A V_{k}=V_{k} T_{k}+v_{k+1} e_{k}^{*}$. Then

$$
Y^{*} X=W_{k}^{*} V_{k}+z w^{*} V_{k}=I
$$

and

$$
Y^{*} A X=T_{k}+\left(W_{k}^{*} v_{k+1}+z w^{*} v_{k+1}\right) e_{k}^{*}=T_{k}+z e_{k}^{*} .
$$

Since the first $k-\ell$ columns of $Y$ are the same as those of $W_{k}$,

$$
\operatorname{span}(Y) \supseteq \operatorname{span}\left(W_{k-\ell}\right)=\mathcal{K}_{k-\ell}\left(A^{*}, l\right) .
$$

Still $\operatorname{span}(X)=\mathcal{K}_{k}(A, b)$. By Theorem 3.3, we have (3.16). The proof for the case $w_{k+1} \neq 0$ is similar.

\section{Structure-preserving moment-matching theorem}

We now extend the moment-matching theorems presented in the previous section to preserve substructures in the system matrices $L, G, C$ and $B$. Specifically, suppose the matrices $L, G, C, B$ in the transfer function (1.1) have some natural partitioning that is derived from, e.g., the physical layout of a VLSI circuit or a structural dynamical system:

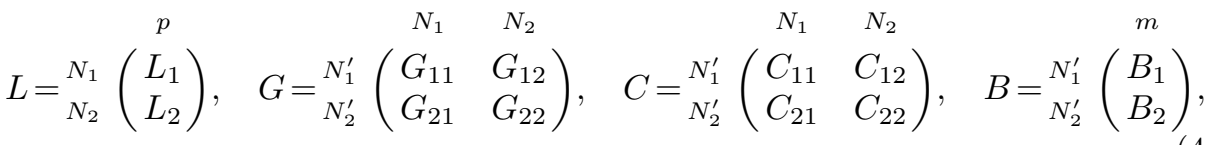

where $N_{1}^{\prime}+N_{2}^{\prime}=N_{1}+N_{2}=N$. We wish the reduced system to inherit the same structure; that is, $L_{\mathrm{R}}, G_{\mathrm{R}}, C_{\mathrm{R}}$ and $B_{\mathrm{R}}$ should be partitioned so that

$$
L_{\mathrm{R}}={ }_{n_{2}}^{n_{1}}\left(\begin{array}{c}
L_{\mathrm{R} 1} \\
L_{\mathrm{R} 2}
\end{array}\right), G_{\mathrm{R}}={ }_{n_{2}^{\prime}}^{n_{1}^{\prime}}\left(\begin{array}{cc}
n_{1} & n_{2} \\
G_{\mathrm{R} 11} & G_{\mathrm{R} 12} \\
G_{\mathrm{R} 21} & G_{\mathrm{R} 22}
\end{array}\right), C_{\mathrm{R}}={ }_{n_{2}^{\prime}}^{n_{1}^{\prime}}\left(\begin{array}{cc}
n_{\mathrm{R} 11} & C_{\mathrm{R} 12} \\
C_{\mathrm{R} 21} & C_{\mathrm{R} 22}
\end{array}\right), B_{\mathrm{R}}={ }_{n_{2}^{\prime}}^{n_{1}^{\prime}}\left(\begin{array}{c}
m \\
B_{\mathrm{R} 1} \\
B_{\mathrm{R} 2}
\end{array}\right),
$$

that each sub-block is a direct reduction from the corresponding sub-block in the original system, e.g., $G_{\mathrm{R} 11}$ from $G_{11}$, where $n_{1}+n_{2}=n_{1}^{\prime}+n_{2}^{\prime}$. In the formulation (1.4) for the reduced system, this can be accomplished by picking

$$
X={ }_{N_{2}}^{N_{1}}\left(\begin{array}{cc}
n_{1} & n_{2} \\
X_{1} & 0 \\
0 & X_{2}
\end{array}\right), \quad Y={ }_{N_{2}^{\prime}}^{N_{1}^{\prime}}\left(\begin{array}{cc}
n_{1}^{\prime} & n_{2}^{\prime} \\
Y_{1} & 0 \\
0 & Y_{2}
\end{array}\right),
$$


such that $\operatorname{rank}\left(X_{j}\right)=n_{j}, \operatorname{rank}\left(Y_{i}\right)=n_{i}^{\prime}$. Then the submatrices of the coefficient matrices $L_{\mathrm{R}}, G_{\mathrm{R}}, C_{\mathrm{R}}$ and $B_{\mathrm{R}}$ of the reduced system are given by

$$
L_{\mathrm{R} j}=X_{j}^{*} L_{j}, G_{\mathrm{R} i j}=Y_{i}^{*} G_{i j} X_{j}, C_{\mathrm{R} i j}=Y_{i}^{*} C_{i j} X_{j}, B_{\mathrm{R} i}=Y_{i}^{*} B_{i} .
$$

A reduction as in (4.4) is conceivably useful for the system matrices with meaningful substructures. For example, for the time-domain modified nodal analysis (MNA) circuit equations targeted by PRIMA [38] and SyMPVL [21], system matrices have the following natural partitioning (adopting the formulation in [21])

$$
G=\left(\begin{array}{cc}
G_{11} & G_{12} \\
G_{12}^{*} & 0
\end{array}\right), C=\left(\begin{array}{cc}
C_{11} & 0 \\
0 & -C_{22}
\end{array}\right), G_{11}^{*}=G_{11}, C_{i i}^{*}=C_{i i}, L=B,
$$

where $G$ and $C$ are the conductance and susceptance matrices; $G_{11}, C_{11}$, and $C_{22}$ are the matrices that contain the stamps for resistors, capacitors, and inductors, respectively; $G_{12}$ 's entries are either 1 or -1 or 0 , representing the current variables in Kirchhoff's current law equations. Accordingly $B=L$ has a natural partitioning, too. As stated in [38], if the original circuit is composed of passive linear elements only, $C_{i i}$ and $G_{11}$ are all (real) symmetric nonnegative definite. Using reduction (4.4) with $Y=X$, all these substructures will be preserved, except that the entries of $G_{\mathrm{R} 12}$ could be numbers other than 1 or -1 or 0 . Passivity of the system is preserved for the same reason as PRIMA [38].

For the sake of moment-matching, Theorem 3.3 remains true here. In terms of finding $X$ and $Y$ as defined in (4.3), we can use the following generic algorithm which produces $Z={ }_{N_{2}}^{N_{1}}\left(\begin{array}{cc}n_{1} & n_{2} \\ Z_{1} & 0 \\ 0 & Z_{2}\end{array}\right)$ from any given $\widetilde{Z}={ }_{N_{2}}^{N_{1}}\left(\begin{array}{l}\widetilde{Z}_{1} \\ \widetilde{Z}_{2}\end{array}\right)$ such that $\operatorname{span}(Z) \supseteq$ $\operatorname{span}(\widetilde{Z})$.

Algorithm 4.1. From given $\widetilde{Z}$ to $Z$ :

1. Compute $Z_{i} \in \mathbb{C}^{N_{i} \times n_{i}}$ having full column rank such that $\operatorname{span}\left(Z_{i}\right) \supseteq \operatorname{span}\left(\widetilde{Z}_{i}\right)$;

2. Output $Z=\left(\begin{array}{cc}Z_{1} & 0 \\ 0 & Z_{2}\end{array}\right)$.

There are a variety of ways to realize Step 1: Rank revealing QR decompositions, modified Gram-Schmit process, or singular value decompositions [11, 15, 26]. For maximum efficiency, one should make $Z_{i}$ have as few columns as one can. Notice the smallest possible number is $\operatorname{rank}\left(\widetilde{Z}_{i}\right)$, but one may have to add a few more columns to make sure the total number of columns in all $X_{i}$ and that in all $Y_{i}$ are the same, as required by Item 3 of Theorem 4.1 below.

THEOREM 4.1. Let $X$ be the output of Algorithm 4.1 with input $\widetilde{X}$, and likewise let $Y$ be the output with input $\widetilde{Y}$. Assume that $G_{\mathrm{R}}$ is nonsingular (and thus the total number of columns in all $X_{i}$ and that in all $Y_{i}$ must be the same).

1. If $\mathcal{K}_{k}\left(G^{-1} C, G^{-1} B\right) \subseteq \operatorname{span}(\widetilde{X})$ and $Y=X$, then $M_{i}=M_{\mathrm{R} i}$ for $0 \leq i \leq k-1$.

2. If $G$ and $C$ are Hermitian, and if $\mathcal{K}_{k}\left(G^{-1} C, G^{-1}(B L)\right) \subseteq \operatorname{span}(\widetilde{X})$ and $Y=$ $X$, then $M_{i}=M_{\mathrm{R} i}$ for $0 \leq i \leq 2 k-1$.

3. If $\mathcal{K}_{k}\left(G^{-1} C, G^{-1} B\right) \subseteq \operatorname{span}(\widetilde{X})$ and $\mathcal{K}_{r}\left(G^{-*} C^{*}, G^{-*} L\right) \subseteq \operatorname{span}(\widetilde{Y})$, then $M_{i}=$ $M_{\mathrm{R} i}$ for $0 \leq i \leq k+r-1$.

Proof. It is a consequence of Theorem 3.3 and Algorithm 4.1.

Remark 4.1. A natural way to compute $\widetilde{X}$ (and $\widetilde{Y}$ ) is to run a (block) Arnoldi process; but it can also be computed by a modified Arnoldi sub-orthogonalization process to be published elsewhere [33]. 
REMARK 4.2. Analogously to Corollary 3.5, Theorem 4.1 remains valid if $G, M_{i}$, and $M_{\mathrm{R} i}$ are replaced by $G\left(s_{0}\right), M_{i}\left(s_{0}\right)$, and $M_{\mathrm{R} i}\left(s_{0}\right)$, respectively.

Sample implementations to realize the three corresponding statements of Theorem 4.1 are given below, where strAMR stands for structural preserving Arnoldi-type model reduction, while the "L" in strLMR stands for Lanczos-type. Notice that Algorithm 4.4 (strLMR) is essentially a two-sided Arnoldi process, and compared to Algorithm 4.3, it has an advantage of producing a (much) smaller reduced model for the same number of matched moments. But there is a tradeoff, too, namely Algorithm 4.4 does not preserve the symmetry in $G$ and $C$ as Algorithm 4.3 does.

Algorithm 4.2. $\operatorname{strAMR}$ - Sample Implementation:

Given $L, G, C, B$ as in (4.1) and expansion point $s_{0}$.

1. $\widehat{G}=G+s_{0} C$; solve $\widehat{G} \widehat{Q}=B$ for $\widehat{Q}$;

2. $Q_{1}=\operatorname{orth}(\widehat{Q})$ : an orthonormal basis matrix for $\operatorname{span}\left(\widehat{G}^{-1} B\right)$;

3. Arnoldi process computes $\widetilde{X}$ :

For $j=1$ to $k-1$ do

Solve $\widehat{G} \widehat{Q}=C Q_{j}$ for $\widehat{Q}$;

For $i=1$ to $j$ do

EndFor

$$
\widehat{Q}=\widehat{Q}-Q_{i}\left(Q_{i}^{*} \widehat{Q}\right)
$$

$Q_{j+1}=\operatorname{orth}(\widehat{Q})$;

EndFor

$$
\tilde{X}=\left(\begin{array}{llll}
Q_{1} & Q_{2} & \cdots & Q_{k}
\end{array}\right) \text { partitioned as } \tilde{X}={ }_{N_{2}}^{N_{1}}\left(\begin{array}{c}
\tilde{X}_{1} \\
\widetilde{X}_{2}
\end{array}\right) ;
$$

4. $X_{1}=\operatorname{orth}\left(\widetilde{X}_{1}\right) ; X_{2}=\operatorname{orth}\left(\widetilde{X}_{2}\right) ; Y_{i}=X_{i}$;

5. Compute nonzero blocks of $L_{\mathrm{R}}, G_{\mathrm{R}}, C_{\mathrm{R}}$, and $B_{\mathrm{R}}$, as in (4.4);

6. Evaluate the reduced $H_{\mathrm{R}}(s)$ as needed.

Algorithm 4.3. strAMR II- Sample Implementation:

Given $L, G, C, B$ as in (4.1) with Hermitian $G$ and $C$ and expansion point $s_{0}$. Replace $B$ in Step 1 of Algorithm 4.2 by $(B L)$.

Algorithm 4.4. strLMR - Sample Implementation:

Given $L, G, C, B$ as in (4.1) and expansion point $s_{0}$.

1. $\widehat{G}=G+s_{0} C$;

2. solve $\widehat{G} \widehat{Q}=B$ for $\widehat{Q}$;

3. solve $\widehat{G}^{*} \widehat{P}=L$ for $\widehat{P}$;

4. $Q_{1}=\operatorname{orth}(\widehat{Q})$ : a basis matrix for $\operatorname{span}\left(\widehat{G}^{-1} B\right)$;

5. $P_{1}=\operatorname{orth}(\widehat{P})$ : a basis matrix for $\operatorname{span}\left(\widehat{G}^{-*} L\right)$;

6. Arnoldi process computes $\widetilde{X}$ and $\widetilde{Y}$ :

For $j=1$ to $k-1$ do

Solve $\widehat{G} \widehat{Q}=C Q_{j}$ for $\widehat{Q}$

Solve $\widehat{G}^{*} \widehat{P}=C^{*} P_{j}$ for $\widehat{P}$;

For $i=1$ to $j$ do

$$
\begin{aligned}
& \widehat{Q}=\widehat{Q}-Q_{i}\left(Q_{i}^{*} \widehat{Q}\right) ; \\
& \widehat{P}=\widehat{P}-P_{i}\left(P_{i}^{*} \widehat{P}\right) ;
\end{aligned}
$$

EndFor 


$$
\begin{aligned}
Q_{j+1} & =\operatorname{orth}(\widehat{Q}) ; \\
P_{j+1} & =\operatorname{orth}(\widehat{P}) ; \\
\text { EndFor } &
\end{aligned}
$$$$
\widetilde{X}=\left(\begin{array}{llll}
Q_{1} & Q_{2} & \cdots & Q_{k}
\end{array}\right) ; \tilde{Y}=\left(\begin{array}{llll}
P_{1} & P_{2} & \cdots & P_{k}
\end{array}\right) \text {; }
$$$$
\text { partitioned as } \widetilde{X}={ }_{N_{2}}^{N_{1}}\left(\begin{array}{c}
\widetilde{X}_{1} \\
\widetilde{X}_{2}
\end{array}\right), \widetilde{Y}={ }_{N_{2}}^{N_{1}}\left(\begin{array}{c}
\widetilde{Y}_{1} \\
\widetilde{Y}_{2}
\end{array}\right)
$$

7. $X_{1}=\operatorname{orth}\left(\widetilde{X}_{1}\right) ; X_{2}=\operatorname{orth}\left(\widetilde{X}_{2}\right)$;

8. $Y_{1}=\operatorname{orth}\left(\widetilde{Y}_{1}\right) ; Y_{2}=\operatorname{orth}\left(\widetilde{Y}_{2}\right) ;$

9. Make sure that the total number of columns in $X_{1}$ and $X_{2}$ is the same as that in $Y_{1}$ and $Y_{2}$; if not, add random columns to the pair with less columns;

10. Compute nonzero blocks of $L_{\mathrm{R}}, G_{\mathrm{R}}, C_{\mathrm{R}}$, and $B_{\mathrm{R}}$, as in (4.4);

11. Evaluate the reduced $H_{\mathrm{R}}(s)$ as needed.

REMARK 4.3. Results in this section are, naturally, extensible to partitions other than 2-by-2. Here is an outline. Suppose we have partitionings

$$
L={ }_{N_{d}}^{N_{2}}\left(\begin{array}{c}
L_{1} \\
L_{2} \\
\vdots \\
L_{d}
\end{array}\right), \quad G={ }_{N_{c}^{\prime}}^{N_{1}^{\prime}}\left(\begin{array}{cccc}
N_{1} & N_{2} & & N_{d} \\
G_{11} & G_{12} & \cdots & G_{1 d} \\
G_{21} & G_{22} & \cdots & G_{2 d} \\
\vdots & \vdots & \ddots & \vdots \\
G_{c 1} & G_{c 2} & \cdots & G_{c d}
\end{array}\right), \quad B={ }_{N_{2}^{\prime}}\left(\begin{array}{c}
B_{1} \\
B_{2} \\
\vdots \\
B_{c}
\end{array}\right),
$$

and $C$ partitioned in the same way as $G$, where $\sum_{j=1}^{c} N_{j}^{\prime}=\sum_{i=1}^{d} N_{i}=N$. To reduce them block-wise to

$$
L_{\mathrm{R}}={ }_{n_{2}}^{n_{1}}\left(\begin{array}{c}
L_{\mathrm{R} 1} \\
L_{\mathrm{R} 2} \\
\vdots \\
L_{\mathrm{R} d}
\end{array}\right), \quad G_{\mathrm{R}}={ }_{n_{c}^{\prime}}^{n_{1}^{\prime}}\left(\begin{array}{cccc}
n_{1} & n_{2} & & n_{d} \\
G_{\mathrm{R} 11} & G_{\mathrm{R} 12} & \cdots & G_{\mathrm{R} 1 d} \\
\vdots & \vdots & \ddots & \vdots \\
G_{\mathrm{R} c 1} & G_{\mathrm{R} 22} & \cdots & G_{\mathrm{R} c d}
\end{array}\right), \quad B_{\mathrm{R}}={ }_{n_{2}^{\prime}}^{n_{1}^{\prime}}\left(\begin{array}{c}
B_{\mathrm{R} 1} \\
B_{\mathrm{R} 2} \\
\vdots \\
B_{\mathrm{R} c}
\end{array}\right),
$$

and $C_{\mathrm{R}}$ partitioned in the same way as $G_{\mathrm{R}}$, we shall pick

$$
X={ }_{N_{d}}^{N_{2}}\left(\begin{array}{cccc}
N_{1} & n_{2} & & n_{d} \\
& X_{2} & & \\
& & \ddots & \\
& & & X_{d}
\end{array}\right), \quad Y={ }_{N_{c}^{\prime}}^{N_{2}^{\prime}}\left(\begin{array}{ccccc}
Y_{1} & & & \\
& Y_{2} & & \\
& & \ddots & \\
& & & Y_{c}
\end{array}\right),
$$

where $\operatorname{rank}\left(X_{j}\right)=n_{j}, \operatorname{rank}\left(Y_{i}\right)=n_{i}^{\prime}, \sum_{j=1}^{c} n_{j}^{\prime}=\sum_{i=1}^{d} n_{i}$. Let the definitions in (4.4) remain valid for $1 \leq i \leq c, 1 \leq j \leq d$. Then versions of Algorithm 4.1 and Theorem 4.1 for $c \geq 3$ and/or $d \geq 3$ can be gotten. Detail is omitted.

\section{Structures of Krylov subspaces of block matrices}

The results of this section may be of general interest, and will be applied to linearized second-order dynamical systems in the next section to derive structurepreserving reduced models. The matrices here do not necessarily have anything to do 
with the transfer function. Consider

$$
A={ }_{N_{2}}^{N_{1}}\left(\begin{array}{cc}
N_{1} & N_{2} \\
A_{11} & A_{12} \\
A_{21} & A_{22}
\end{array}\right), \quad B={ }_{N_{2}}^{N_{1}}\left(\begin{array}{c}
m \\
B_{1} \\
B_{2}
\end{array}\right),
$$

where $N_{1}+N_{2}=N$. The following theorem describes the structures in a basis matrix of $\mathcal{K}_{k}(A, B)$ when one of $A_{i j}$ 's is zero.

Theorem 5.1. Let $A$ and $B$ be partitioned as in (5.1), and let $\operatorname{span}(\widetilde{X})=\mathcal{K}_{k}(A, B)$ be partitioned as

$$
\widetilde{X}={ }_{N_{2}}^{N_{1}}\left(\begin{array}{cc}
n_{1}^{\prime} & \widetilde{X}_{11}^{n_{2}^{\prime}} \\
\widetilde{X}_{21} & \widetilde{X}_{22}
\end{array}\right) \equiv_{N_{2}}^{N_{1}}\left(\begin{array}{c}
n_{1}^{\prime}+n_{2}^{\prime} \\
\widetilde{X}_{1} \\
\widetilde{X}_{2}
\end{array}\right)
$$

such that $\operatorname{span}\left(\begin{array}{l}\widetilde{X}_{11} \\ \widetilde{X}_{21}\end{array}\right)=\mathcal{K}_{k-1}(A, B)$, and let $\alpha \neq 0$ be a scalar which may be different at different occurrences. Then

1. If $A_{11}=0$, then $\operatorname{span}\left(\widetilde{X}_{1}\right)=\operatorname{span}\left(B_{1} A_{12} \widetilde{X}_{21}\right) \subseteq \operatorname{span}\left(B_{1} A_{12} \widetilde{X}_{2}\right)$. If in addition $A_{12}=\alpha I$ (and thus $\left.N_{1}=N_{2}\right), \operatorname{span}\left(\widetilde{X}_{1}\right)=\operatorname{span}\left(B_{1} \tilde{X}_{21}\right) \subseteq \operatorname{span}\left(B_{1} \widetilde{X}_{2}\right)$.

2. If $A_{12}=0$, then $\operatorname{span}\left(\widetilde{X}_{1}\right)=\mathcal{K}_{k}\left(A_{11}, B_{1}\right)$.

3. If $A_{21}=0$, then $\operatorname{span}\left(\widetilde{X}_{2}\right)=\mathcal{K}_{k}\left(A_{22}, B_{2}\right)$.

4. If $A_{22}=0$, then $\operatorname{span}\left(\widetilde{X}_{2}\right)=\operatorname{span}\left(B_{2} A_{21} \widetilde{X}_{11}\right) \subseteq \operatorname{span}\left(B_{2} A_{21} \widetilde{X}_{1}\right)$. If in addition $A_{21}=\alpha I$ (and thus $\left.N_{1}=N_{2}\right), \operatorname{span}\left(\widetilde{X}_{2}\right)=\operatorname{span}\left(B_{2} \widetilde{X}_{11}\right) \subseteq \operatorname{span}\left(B_{2} \widetilde{X}_{1}\right)$.

Proof. All claims are consequences of the following observation:

$$
\text { if } \quad A^{i} B=\left(\begin{array}{l}
Z_{1} \\
Z_{2}
\end{array}\right), \text { then } A^{i+1} B=\left(\begin{array}{l}
A_{11} Z_{1}+A_{12} Z_{2} \\
A_{21} Z_{1}+A_{22} Z_{2}
\end{array}\right) .
$$

Then combining the assumption that one of $A_{i j}=0$ will complete the proof.

Item 4 of Theorem 5.1 was implicitly stated in $[49,7,8]$. It gives a relation between $\operatorname{span}\left(\widetilde{X}_{1}\right)$ and $\operatorname{span}\left(\widetilde{X}_{2}\right)$; so does Item 1. It is Item 4 that led to structure-preserving dimension reduction of second-order systems. See Section 6 .

Theorem 5.1 can be extended to block matrices $A$ other than just $2 \times 2$ block matrices. A conceivable case that allows us to derive simple relations among blocks of a basis matrix (conformably partitioned) of Krylov subspaces is when all block rows, except one, of $A$ have only one nonzero block, i.e., if

$$
A={ }_{N_{d}}^{N_{1}}\left(\begin{array}{cccc}
N_{1} & N_{2} & & N_{d} \\
A_{11} & A_{12} & \cdots & A_{1 d} \\
A_{21} & A_{22} & \cdots & A_{2 d} \\
\vdots & \vdots & \ddots & \vdots \\
A_{d 1} & A_{d 2} & \cdots & A_{d d}
\end{array}\right),
$$

then except one block row of $A$, each of the rest block rows has at most one nonzero 
block. Even so there are still many subcases, one of which is

$$
A={ }_{N_{d}}^{N_{1}}\left(\begin{array}{cccc}
N_{1} & N_{2} & & N_{d} \\
A_{11} & A_{12} & \ldots & A_{1 d} \\
A_{21} & 0 & & \\
& \ddots & \ddots & \\
& & A_{d d-1} & 0
\end{array}\right) .
$$

Matrices like this arises from linearization of a polynomial eigenvalue problem [25] in which $A_{21}=A_{32}=\cdots=A_{d d-1}=I$ and $N_{1}=N_{2}=\cdots=N_{d}$. As an example, we shall state a theorem for $A$ having form (5.2).

TheOrem 5.2. Let $A$ be partitioned as in $(5.2)$, and let $\operatorname{span}(\tilde{X})=\mathcal{K}_{k}(A, B)$. Partition $B$ and $\widetilde{X}$ accordingly as

$$
B={ }_{N_{d}}^{N_{2}}\left(\begin{array}{c}
B_{1} \\
B_{2} \\
\vdots \\
B_{d}
\end{array}\right), \quad \tilde{X}={ }_{N_{d}}^{N_{2}}\left(\begin{array}{c}
\widetilde{X}_{1} \\
\widetilde{X}_{2} \\
\vdots \\
\tilde{\widetilde{X}}_{d}
\end{array}\right) .
$$

Then

$$
\operatorname{span}\left(\tilde{X}_{i}\right) \subseteq \operatorname{span}\left(B_{i} A_{i i-1} \tilde{X}_{i-1}\right)
$$

for $2 \leq i \leq d$. If in addition $A_{i-1}=\alpha_{i} I$ for $2 \leq i \leq d$ (and thus $N_{1}=N_{2}=\cdots=N_{d}$ ), then

$$
\operatorname{span}\left(\widetilde{X}_{i}\right) \subseteq \operatorname{span}\left(B_{i} \quad \widetilde{X}_{i-1}\right) \subseteq \operatorname{span}\left(B_{i} \quad B_{i-1} \quad \cdots \quad B_{2} \quad \widetilde{X}_{1}\right)
$$

for $2 \leq i \leq d$.

\section{Structure-preserving model reduction of second-order systems}

In this section, we show how to apply the theory presented in the previous sections to the structure-preserving model reduction of a second-order system. Consider the transfer function of a second-order system

$$
H(s)=\left(V^{*}+s T^{*}\right)\left(s^{2} M+s D+K\right)^{-1} R,
$$

which arises from applying the Laplace transform to the time-invariant MIMO secondorder dynamical system

$$
\left\{\begin{aligned}
M \ddot{q}(t)+D \dot{q}(t)+K q(t) & =R u(t), \\
y(t) & =T^{*} \dot{q}(t)+V^{*} q(t),
\end{aligned}\right.
$$

where $M, D, K \in \mathbb{C}^{N \times N}, R \in \mathbb{C}^{N \times m}, T, V \in \mathbb{C}^{N \times p}$, and $u(t), q(t)$ and $y(t)$ are vectorvalued functions of appropriate dimensions. Notation here is adopted from structural dynamics, where $M, D, K$ are mass, damping, and stiffness matrices and are usually Hermitian, but can be non-Hermitian at times.

It is quite common to deal with $(6.1)$ by a linearization technique to turn it into the form of (1.1). This is done by setting

$$
C=\left(\begin{array}{cc}
D & M \\
W & 0
\end{array}\right), G=\left(\begin{array}{cc}
K & 0 \\
0 & -W
\end{array}\right), L=\left(\begin{array}{l}
V \\
T
\end{array}\right), B=\left(\begin{array}{c}
R \\
0
\end{array}\right)
$$


where $W$ is any $N \times N$ nonsingular matrix, usually taken to be $M$ when $M, D, K$ are Hermitian or simply $I$ otherwise. By now, all existing developments for the transfer function (1.1) can be applied in a straightforward way, but then reduced models likely lose the second-order characteristics, i.e., they may not be turned into the secondorder transfer functions ${ }^{2}$ and consequently the reduced models have little physical significance. To overcome this, Su and Craig [49] made an important observation which is equivalent to Item 4 of Theorem 5.1. Their work has been further developed in $[7,8]$. Similar efforts were made by $[30,36]$ where algorithms are proposed and are provably efficient for so-called low-rank cases. Specifically, in a structure-preserving model reduction method, the transfer function of the reduced second-order system is also of the second-order form

$$
H_{\mathrm{R}}(s)=\left(V_{\mathrm{R}}^{*}+s T_{\mathrm{R}}^{*}\right)\left(s^{2} M_{\mathrm{R}}+s D_{\mathrm{R}}+K_{\mathrm{R}}\right)^{-1} R_{\mathrm{R}},
$$

where

$$
\begin{aligned}
& M_{\mathrm{R}}=Y_{1}^{*} M X_{1}, D_{\mathrm{R}}=Y_{1}^{*} D X_{1}, K_{\mathrm{R}}=Y_{1}^{*} K X_{1}, \\
& V_{\mathrm{R}}=X_{1}^{*} V, T_{\mathrm{R}}=X_{1}^{*} T, R_{\mathrm{R}}=Y_{1}^{*} R,
\end{aligned}
$$

and $X_{1}, Y_{1} \in \mathbb{C}^{N \times n}$ having full column rank. Together with $L, G, C$ and $B$ as defined by (6.2), the transfer functions $H(s)$ and $H_{\mathrm{R}}(s)$ of the original and reduced systems can be written in the following linear forms

$$
H(s)=L^{*}(G+s C)^{-1} B \quad \text { and } \quad H_{\mathrm{R}}(s)=L_{\mathrm{R}}^{*}\left(G_{\mathrm{R}}+s C_{\mathrm{R}}\right)^{-1} B_{\mathrm{R}},
$$

where $L_{\mathrm{R}}=X^{*} L, G_{\mathrm{R}}=Y^{*} G X, C_{\mathrm{R}}=Y^{*} C X$, and $B_{\mathrm{R}}=Y^{*} B$ as in (1.4), and

$$
X={ }_{N}^{N}\left(\begin{array}{cc}
n & n \\
X_{1} & 0 \\
0 & X_{1}
\end{array}\right) \text { and } Y={ }_{N}^{N}\left(\begin{array}{cc}
n & n \\
Y_{1} & 0 \\
0 & Y_{1}
\end{array}\right) .
$$

We now show the moment-matching property of the reduced transfer function $H_{\mathrm{R}}(s)$. Assume that $K$ and $W$ are nonsingular, we have

$$
G^{-1} C=\left(\begin{array}{cc}
K^{-1} D & K^{-1} M \\
-I & 0
\end{array}\right), \quad G^{-*} C^{*}=\left(\begin{array}{cc}
K^{-*} D^{*} & K^{-*} W^{*} \\
-W^{-*} M^{*} & 0
\end{array}\right) .
$$

Both $G^{-1} C$ and $G^{-*} C^{*}$ have a zero block, a condition of Theorem 5.1. Moreover the (2,1)-block of $G^{-1} C$ is $-I$ always, and that of $G^{-*} C^{*}$ can be made $-I$, if $W=M$. In what follows, $X$ and $Y$ are defined as in (6.5) in terms of $X_{1}$ and $Y_{1}$, and $\widetilde{X}$ and $\widetilde{Y}$ are always partitioned as

$$
\widetilde{X}={ }_{N}^{N}\left(\begin{array}{l}
\widetilde{X}_{1} \\
\widetilde{X}_{2}
\end{array}\right) \text { and } \quad \widetilde{Y}={ }_{N}^{N}\left(\begin{array}{c}
\widetilde{Y}_{1} \\
\widetilde{Y}_{2}
\end{array}\right)
$$

\footnotetext{
${ }^{2}$ It is possible to turn a linear system of even dimension into a second-order system. Recently $[37,45]$ and [29] propose two different ways to do that; but in both cases the coefficient matrices of the resulted second-order system cannot be related to the original system in a meaningful way.
} 
Theorem 6.1. Suppose that $M, D$, and $K$ are Hermitian, and $M$ and $K$ are nonsingular. Let integer $k \geq 0, L, G, C$ and $B$ be defined in (6.2) and $W=M$. If

$$
\begin{aligned}
& \mathcal{K}_{k}\left(G^{-1} C, G^{-1}(B L)\right) \subseteq \operatorname{span}(\widetilde{X}), \\
& \operatorname{span}\left(M^{-1} T\right) \subseteq \operatorname{span}\left(X_{1}\right), \\
& \operatorname{span}\left(\widetilde{X}_{1}\right) \subseteq \operatorname{span}\left(X_{1}\right),
\end{aligned}
$$

and $Y=X$, then $H(s)=H_{\mathrm{R}}(s)+\mathcal{O}\left(s^{2 k}\right)$.

Proof. Note that $W=M$ implies that both (2,1)-blocks in (6.6) are $-I$. By (6.8), (6.9), Item 4 of Theorem 5.1, and $B$ and $L$ in (6.2), we have

$$
\operatorname{span}\left(\widetilde{X}_{2}\right) \subseteq \operatorname{span}\left(M^{-1} T \quad \widetilde{X}_{1}\right) \subseteq \operatorname{span}\left(X_{1}\right)
$$

which, together with (6.9), imply $\operatorname{span}(\widetilde{X}) \subseteq \operatorname{span}(X)$, and hence

$$
\mathcal{K}_{k}\left(G^{-1} C, G^{-1} B\right) \subseteq \mathcal{K}_{k}\left(G^{-1} C, G^{-1}(B L)\right) \subseteq \operatorname{span}(\widetilde{X}) \subseteq \operatorname{span}(X) .
$$

Furthermore, since $G$ and $C$ are Hermitian, we have

$\mathcal{K}_{k}\left(G^{-*} C^{*}, G^{-*} L\right)=\mathcal{K}_{k}\left(G^{-1} C, G^{-1} L\right) \subseteq \mathcal{K}_{k}\left(G^{-1} C, G^{-1}(B L)\right) \subseteq \operatorname{span}(\widetilde{X}) \subseteq \operatorname{span}(X)$.

The conclusion of the theorem is now a consequence of Theorem 3.3.

A sample Arnoldi-type implementation is as follows. Another implementation includes the original one of [49]

Algorithm 6.1. qAMR - Sample Implementation: Computing $X_{1}$.

1. Compute $\widehat{X}$ such that $\mathcal{K}_{q}\left(G^{-1} C, G^{-1}(B L) \subseteq \operatorname{span}(\widehat{X})\right.$, by, e.g., Algorithm 4.2;

2. Partition $\widehat{X}={ }_{N}^{N}\left(\begin{array}{c}\widehat{X}_{1} \\ \widehat{X}_{2}\end{array}\right)$;

3. Compute $X_{1}=\operatorname{orth}\left(\left(\widehat{X}_{1} M^{-1} T\right)\right)$.

REMARK 6.1. Su and Craig's algorithm [49], in our notation, essentially first computes $\widetilde{X}$ by a block Arnoldi-like procedure to satisfy

$$
\mathcal{K}_{k}\left(G^{-1} C, G^{-1}\left(\begin{array}{ccc}
R & V & T \\
0 & T & 0
\end{array}\right)\right) \subseteq \operatorname{span}(\widetilde{X}),
$$

and then uses $X_{1}=Y_{1}=\widetilde{X}_{1}$ to define a reduced second-order system as in (6.3). Note that doing so may unnecessarily produce a reduced system larger than it should be because the entire $\left(\begin{array}{c}M^{-1} T \\ 0\end{array}\right)$ participates the Arnoldi process, whereas Theorem 6.1 says only the range of $M^{-1} T$ needs to be included in $X_{1}$. In fact it can be proved that

$$
\operatorname{span}\left(\left(\begin{array}{c}
M^{-1} T \\
0
\end{array}\right), \mathcal{K}_{k}\left(G^{-1} C, G^{-1}(B L)\right)\right) \subseteq \mathcal{K}_{k}\left(G^{-1} C, G^{-1}\left(\begin{array}{ccc}
R & V & T \\
0 & T & 0
\end{array}\right)\right) .
$$

The so-called second-order Arnoldi procedure recently presented in $[7,8]$ can be interpreted as an algorithm to directly compute an orthonormal basis of $\operatorname{span}\left(X_{1}\right)$. 
The theorem below does not assume that $M, D$, and $K$ are Hermitian.

TheOrem 6.2. Suppose that $M$ and $K$ are nonsingular. Let integers $k, r \geq 0, L, G, C$ and $B$ be defined in (6.2) and $W=M$. If

$$
\begin{aligned}
& \mathcal{K}_{k}\left(G^{-1} C, G^{-1} B\right) \subseteq \operatorname{span}(\tilde{X}), \\
& \mathcal{K}_{r}\left(G^{-*} C^{*}, G^{-*} L\right) \subseteq \operatorname{span}(\widetilde{Y}), \\
& \operatorname{span}\left(\widetilde{X}_{1}\right) \subseteq \operatorname{span}\left(X_{1}\right), \\
& \operatorname{span}\left(M^{-1} T\right) \subseteq \operatorname{span}\left(Y_{1}\right), \\
& \operatorname{span}\left(\widetilde{Y}_{1}\right) \subseteq \operatorname{span}\left(Y_{1}\right),
\end{aligned}
$$

then $H(s)=H_{\mathrm{R}}(s)+\mathcal{O}\left(s^{k+r}\right)$.

Proof. Note that $W=M$ implies that both (2,1)-blocks in (6.6) are $-I$. By Item 4 of Theorem 5.1 , the $B$ in (6.2), we have

$$
\operatorname{span}\left(\widetilde{X}_{2}\right) \subseteq \operatorname{span}\left(\widetilde{X}_{1}\right) .
$$

Therefore, by (6.10) and (6.12), we have

$$
\mathcal{K}_{k}\left(G^{-1} C, G^{-1} B\right) \subseteq \operatorname{span}(\tilde{X}) \subseteq \operatorname{span}(X) .
$$

On the other hand, by (6.13) and Item 4 of Theorem 5.1, we have

$$
\operatorname{span}\left(\widetilde{Y}_{2}\right) \subseteq \operatorname{span}\left(M^{-1} T \tilde{Y}_{1}\right) \subseteq \operatorname{span}\left(Y_{1}\right)
$$

which, together with (6.11) and (6.14), imply

$$
\mathcal{K}_{r}\left(G^{-*} C^{*}, G^{-*} L\right) \subseteq \operatorname{span}(Y) .
$$

The conclusion is now a consequence of Theorem 3.3.

REMARK 6.2. The idea in this section is naturally extensible to transfer functions of higher degree of the form

$$
H(s)=\left(\sum_{i=0}^{d-1} s^{i} V_{i}\right)\left(\sum_{i=0}^{d} s^{i} A_{i}\right)^{-1} R
$$

thanks to Theorem 5.2. Detail is omitted. Other studies in high-order transfer functions include [14, 17, 47, 48].

\section{Numerical Examples}

The first example is taken from [46]. Here $N=256$, the structure of $G$ and $C$ are as in Figure 7.1, $N_{i}^{\prime}=N_{i}=128(i=1,2), p=m=1$, and $L$ and $B$ are randomly chosen. We compare the approximate accuracy of the "structurally reduced" models by Algorithm 4.2 as proposed against otherwise "generally reduced" ones, i.e., Algorithm 4.2 without Step 4 (and therefore $X=\widetilde{X}$ ). Figure 7.2 plots the values of the original and reduced transfer functions and the relative errors of the reduced functions, where $Y=X$ and $\operatorname{span}(X) \supset \mathcal{K}_{20}\left(G^{-1} C, G^{-1} B\right)$. It clearly shows that the structurally reduced model is more accurate in the long range of frequency.

It is natural to wonder whether incorrect structural partitioning would make any difference. Indeed it does. Supposedly we take $N_{1}^{\prime}=N_{1}=128+20$ and $N_{2}^{\prime}=N_{2}=$ 

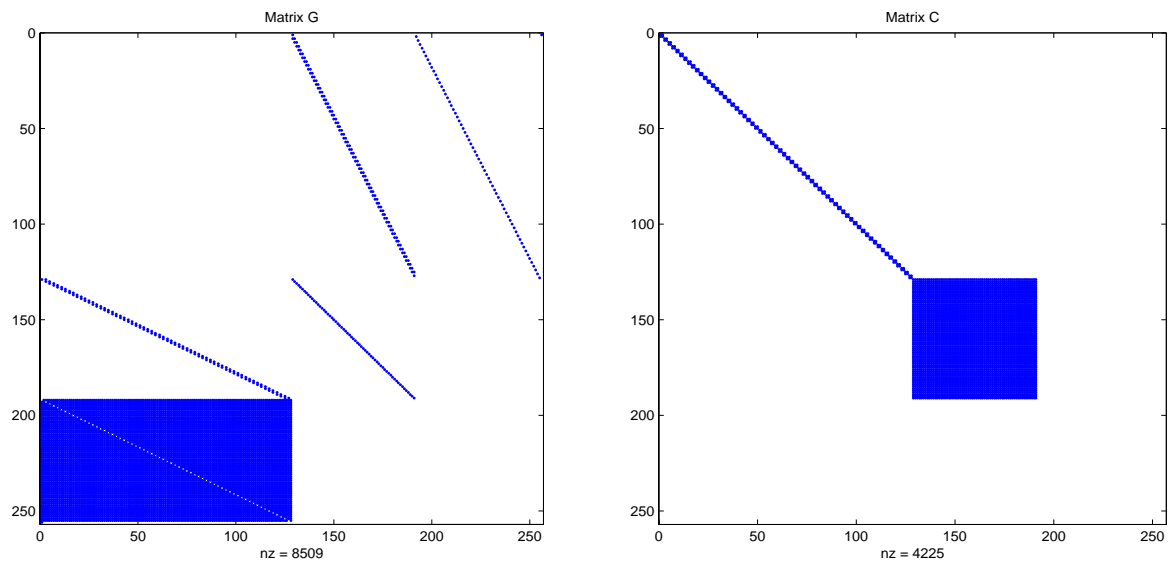

FIG. 7.1. Block Structure of $G$ (left) and C (right)
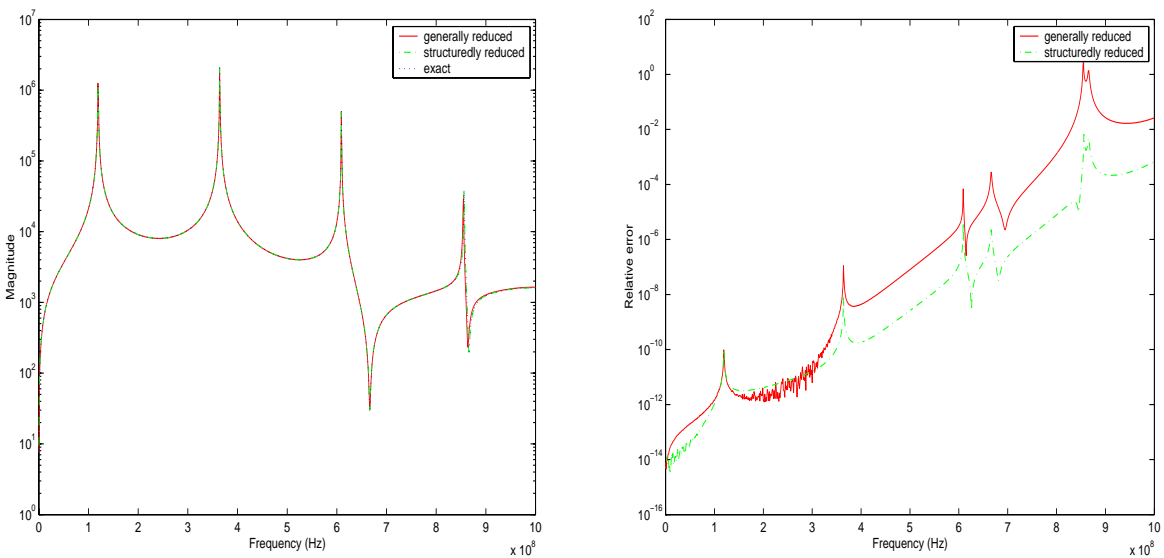

FIG. 7.2. Transfer functions (left) and relative errors (right)

$128-20$. Figure 7.3 plots the same things as Figure 7.2, except with this new partition, where again $Y=X$ and $\operatorname{span}(X) \supset \mathcal{K}_{20}\left(G^{-1} C, G^{-1} B\right)$. This figure shows that improper partitioning can degrade accuracy. But more than that, for this partitioning "structural reduction" is less accurate than the "general reduction" which is quite counter-intuitive and surprising because $\operatorname{span}(X)$ with some partitioning includes $\operatorname{span}(X)$ without any partitioning, and thus a reduction with partitioning should do at least just as well as one without in terms of accuracy - further studies needed.

Next example is the second-order system from $[4, \S 3.3]: N=400, p=m=1, T=0$, and $V=R$ randomly chosen. Figure 7.4 plots the values of the original and reduced transfer functions and relative errors, where "quadratically reduced" refers to (6.3) with (6.4) and $X_{1}$ by, e.g., Algorithm 6.1, and "linearly reduced" refers to (1.3) and (1.4) through linearization (6.2) with $Y=X(=\widehat{X}$ in Algorithm 6.1 without Step 3).

\section{Concluding remarks}

We presented a Krylov subspace based projection formulation for structurepreserving model reduction of a MIMO dynamical system prescribed by its transfer 

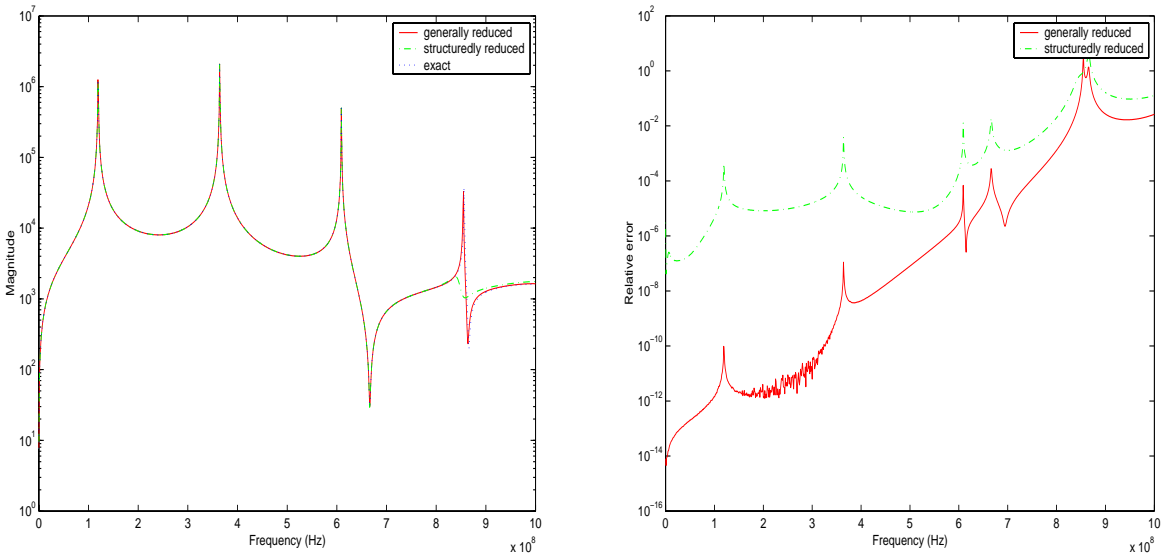

FIG. 7.3. Transfer functions (left) and relative errors (right); Preserving incorrect structure can lead to less accurate approximations.
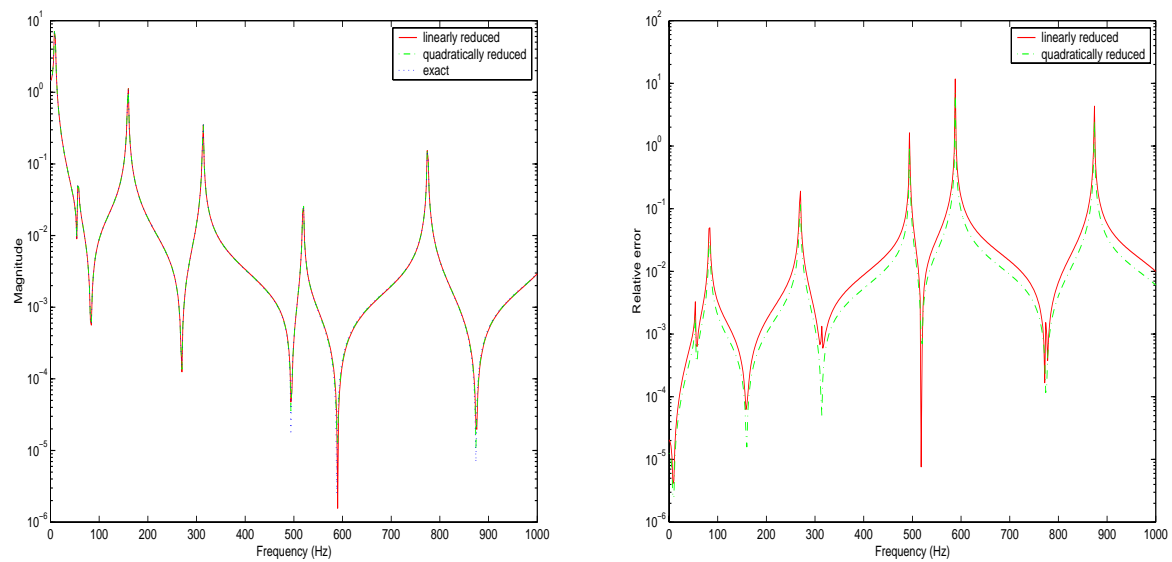

FIG. 7.4. Transfer functions (left) and relative errors (right): a second-order example

function $H(s)=L^{*}(G+s C)^{-1} B$. The reduced-order system preserves substructures of importance in the coefficient matrices $L, G, C$ and $B$. Such a projection formulation provides a simple interpretation to many existing methods, meanwhile it also has extreme flexibility to exploit the structures of coefficient matrices $L, G, C$ and $B$ and the associated Krylov subspaces. We can generalize the work presented in $[7,8]$ to develop new Arnoldi-like processes that only orthogonalize the prescribed portion of all basis vectors as opposed to whole vectors. Such new processes can be designed as one way to numerically realize the idea in the general framework as we discussed in this paper.

The work of Su and Craig [49] has spawned several recent research papers on model reduction of second-order systems and quadratic eigenvalue problems, including $[7,8,18,50]$. But the attempt to preserve meaningful substructures as in (4.1) - (4.4) for any general linear systems, not necessarily from linearizing a second-order system, appears to be conceived first by [33, 34]. 
Acknowledgment. Li and Bai are supported in part by NSF CAREER award under grant CCR-9875201 and NSF ITR grant ACI-0220104, respectively.

\section{REFERENCES}

[1] A. C. Antoulas, D. C. Sorensen and S. Gugercin, A survey of model reduction methods for largescale systems, Structured Matrices in Mathematics, Computer Science, and Engineering I: Proceedings of an AMS-IMS-SIAM joint summer research conference, University of Colorado, Boulder, June 27-July 1, 1999, V. Olshevsky, ed., of Contemporary Mathematics, American Mathematical Society, Providence, Rhode Island, 280, 193-219, 2001.

[2] W. E. Arnoldi, The principle of minimized iterations in the solution of the matrix eigenvalue problem, Quarterly of Applied Math., 9, 17-29, 1951.

[3] Z. Bai, Error analysis of the Lanczos algorithm for the nonsymmetric eigenvalue problem, Mathematics of Computation, 62, 209-226, 1994.

[4] Z. Bai, Krylov subspace techniques for reduced-order modeling of large-scale dynamical systems, Applied Numerical Mathematics, 43, 9-44, 2002.

[5] Z. Bai, D. Bindel, J. Clark, J. Demmel, K. S. J. Pister and N. Zhou, New numerical techniques and tools in SUGAR for 3D MEMS simulation, in Technical Proceedings of the Fourth International Conference on Modeling and Simulation of Microsystems, 31-34, 2000.

[6] Z. Bai and R. Freund, A partial Padé-via-Lanczos method for reduced-order modeling, Linear Algebra and its Applications, 332-334, 139-164, 2001.

[7] Z. Bai and Y.Su, SOAR: A second-order Arnoldi method for the solution of the quadratic eigenvalue problem, Computer Science Technical Report CSE-2003-21, University of California, Davis, California, 2003, Submitted to SIMAX.

[8] Z. Bai and Y. Su, Dimension reduction of second-order dynamical systems via a second-order Arnoldi method, Computer Science Technical Report CSE-2004-1, University of California, Davis, California, 2004.

[9] Z. Bai and Q. Ye, Error estimation of the Padé approximation of transfer functions via the Lanczos process, Electronic Transactions on Numerical Analysis, 7, 1-17, 1998.

[10] M. J. Balas, Trends in large space structure control theory: fondest theory, wildest dreams, IEEE Trans. Automat. Control, AC-27, 522-535, 1982.

[11] A. Björck, Numerical Methods for Least Squares Problems, SIAM, Philadelphia, 1996.

[12] J. V. Clark, N. Zhou, D. Bindel, L. Schenato, W. Wu, J. Demmel and K. S. J. Pister, 3D MEMS simulation using modified nodal analysis, in Proceedings of Microscale Systems: Mechanics and Measurements Symposium, 68-75, 2000.

[13] Jr. R. R. Craig, Structural Dynamics: An Introduction to Computer Methods, John Wiley \& Sons, 1981.

[14] J. Cullum, A. Ruehli and T. Zhang, A method for reduced-order modeling and simulation of large interconnect circuits and its application to PEEC models with retardation, IEEE Trans. Circuits and Systems - II, 47, 261-273, 2000.

[15] J. Demmel, Applied Numerical Linear Algebra, SIAM, Philadelphia, 1997.

[16] P. Feldman and R. W. Freund, Efficient linear circuit analysis by Padé approximation via the Lanczos process, IEEE Trans. Computer-Aided Design, 14, 639-649, 1995.

[17] P. Feldmann and R. W. Freund, Circuit noise evaluation by Padé approximation based modelreduction techniques, in Technical Digest of the 1997 IEEE/ACM International Conference on Computer-Aided Design, IEEE Computer Society Press, 132-138, 1997.

[18] R. W. Freund, Pade-type reduced-order modeling of higher-order systems, Presentation at Oberwolfach Mini-Workshop on Dimensional Reduction of Large-Scale Systems, October, 2003.

[19] R. W. Freund, Krylov-subspace methods for reduced-order modeling in circuit simulation, Journal of Computational and Applied Mathematics, 123, 395-421, 2000.

[20] R. W. Freund, Model reduction methods based on Krylov subspaces, Acta Numerica, 12, 267-319, 2003.

[21] R. W. Freund and P. Feldmann, The SyMPVL algorithm and its applications to interconnect simulation, in Proc. 1997 International Conference on Simulation of Semiconductor Processes and Devices, Piscataway, New Jersey, IEEE, 113-116, 1997.

[22] R. W. Freund, M. H. Gutknecht and N. M. Nachtigal, An implementation of the look-ahead Lanczos algorithm for non-Hermitian matrices, SIAM J. Sci. Comput., 14, 137-158, 1993. 
[23] K. Gallivan, E. Grimme and P. Van Dooren, Asymptotic waveform evaluation via a Lanczos method, Appl. Math. Lett., 7, 75-80, 1994.

[24] S. D. Garvey, Z. Chen, M. I. Friswell and U. Prells, Model reduction using structure-preserving transformations, in Proceedings of the International Modal Analysis Conference IMAC XXI, Kissimmee, Florida, Feb., 361-377, 2003.

[25] I. Gohberg, P. Lancaster and L. Rodman, Matrix Polynomials, Academic Press, New York, 1982.

[26] G. H. Golub and C. F. Van Loan, Matrix Computations, Johns Hopkins University Press, Baltimore, Maryland, 3rd ed., 1996.

[27] W. B. Gragg, Matrix interpretations and applications of the continued fraction algorithm, Rocky Mountain J. Math., 4, 213-225, 1974.

[28] E. J. Grimme, Krylov Projection Methods For Model Reduction, PhD thesis, University of Illinois at Urbana-Champaign, Urbana, Illinois, 1997.

[29] L. Hoffnung, Subspace Projection Methods for the Quadratic Eigenvalue Problem, PhD thesis, University of Kentucky, Lexington, KY, August 2004.

[30] L. Hoffnung, R. C. Li and Q. Ye, Krylov type subspace methods for matrix polynomials, Linear Algebra and its Applications, 2003, to appear.

[31] T. Kato, Perturbation Theory for Linear Operators, Springer-Verlag, Berlin, 2nd ed., 1970.

[32] C. Lanczos, An iteration method for the solution of the eigenvalue problem of linear differential and integral operators, Journal of Research of the National Bureau of Standards, 45, 255$282,1950$.

[33] R. C. Li, Structural preserving model reductions, Technical Report 2004-02, Department of Mathematics, University of Kentucky, January 2004. Avaliable at http://www.ms.uky.edu / math/MAreport/.

[34] R. C. Li and Z. Bai, Structure-preserving model reductions using a Krylov subspace projection formulation, Tech. Report CSE-2004-24, Department of Computer Science, University of California, Davis, March 2004.

[35] R. C. Li and Z. Bai, Structure-preserving model reduction, in PARA'04 State-of-the-Art in Scientific Computing, June, 20-23, 2004. J. Dongarra, K. Madsen, and J. Wasniewski, eds., Lecture Notes in Computer Science, Springer, 2005, to appear.

[36] R. C. Li and Q. Ye, A Krylov subspace method for quadratic matrix polynomials with application to constrained least squares problems, SIAM J. Matrix Anal. Appl., 25, 405-428, 2003.

[37] D. Meyer and S. Srinivasan, Balancing and model reduction for second-order form linear systems, IEEE Transactions on Automatic Control, 41, 1632-1644, 1996.

[38] A. Odabasioglu, M. Celik and L. T. Pileggi, PRIMA: passive reduced-order interconnect macromodeling algorithm, IEEE Transactions on Computer-Aided Design of Integrated Circuits and Systems, 17, 645-654, 1998.

[39] B. N. Parlett, Reduction to tridiagonal form and minimal realization, SIAM Journal on Matrix Analysis and Applications, 13, 567-593, 1992.

[40] B. N. Parlett, D. R. Taylor and Z. A. Liu, A look-ahead Lanczos algorithm for unsymmetric matrices, Mathematics of Computation, 44, 105-124, 1985.

[41] L. T. Pillage and R. A. Rohrer, Asymptotic waveform evaluation for timing analysis, IEEE Transactions on Computer-Aided Design of Integrated Circuits and Systems, 9, 352-366, 1990

[42] D. Ramaswamy and J. White, Automatic generation of small-signal dynamic macromodels from 3-D simulation, in Technical Proceedings of the Fourth International Conference on Modeling and Simulation of Microsystems, 27-30, 2000.

[43] A. Ruhe, Rational Krylov sequence methods for eigenvalue computation, Linear Algebra and its Applications, 58, 391-405, 1984.

[44] A. Ruhe, Rational Krylov algorithms for nonsymmetric eigenvalue problems. II. matrix pairs, Linear Algebra and its Applications, 197-198, 283-295, 1994.

[45] B. Salimbahrami and B. Lohmann, Structure preserving order reduction of large scale second order systems, in Proceeding of 10th IFAC/IFORS/IMACS/IFIP Symposium on Large Scale Systems: Theory and Applications, Osaka, Japan, July, 245-250, 2004.

[46] R. D. Slone, A computationally efficient method for solving electromagnetic interconnect problems: the Padé approximation via the Lanczos process with an error bound, master's thesis, University of Kentucky, Lexington, KY, 1997. 
[47] R. D. Slone, Fast frequency sweep model order reduction of polynomial matrix equations resulting from finite element discretization, $\mathrm{PhD}$ thesis, Ohio State University, Columbus, $\mathrm{OH}$, 2002.

[48] R. D. Slone, R. Lee and J. F. Lee, Broadband model order reduction of polynomial matrix equations using single point well-conditioned asymptotic waveform evaluation: derivation and theory, Inter. J. Numer. Methods in Eng., 2002, submitted.

[49] T. J. Su and R. R. Craig, Model reduction and control of flexible structures using Krylov vectors, J. Guidance, Control, and Dynamics, 14, 260-267, 1991.

[50] A. Vandendorpe and P. Van Dooren, Krylov techniques for model reduction of second-order systems, Unpublished Note, March 2, 2004.

[51] C. E. Villemagne and R. E. Skelton, Model reduction using a projection formulation, Int. J. Control, 46, 2141-2169, 1987.

[52] T. Wittig, I. Munteanu, R. Schuhmann and T. Weiland, Two-step Lanczos algorithm for model order reduction, IEEE Trans. Magn., 38, 673-676, 2002.

[53] Q. Ye, A convergence analysis for nonsymmetric Lanczos algorithms, Mathematics of Computation, 56, 677-691, 1991.

[54] Q. Ye, A breakdown-free variation of the nonsymmetric Lanczos algorithms, Mathematics of Computation, 62, 179-207, 1994.

[55] H. Zheng and L. Pileggi, Robust and passive model order reduction for circuits constaining susceptance elements, in Tech. Dig. 2002 IEEE/ACM International Conference on ComputerAided Design, IEEE, 2002. 